\title{
Treatment seeking behavior and related delays by pulmonary tuberculosis patients in E-ward of Mumbai Municipal Corporation, India
}

Background: Treatment delays increase adverse treatment outcome of tuberculosis (TB). It is necessary to identify reasons behind such delays in different phases of anti-TB treatment (ATT). Objective: To study treatment seeking behavior (TSB) and risk factors for treatment delays among TB patients. Materials and Methods: New smear positive (NSP) pulmonary TB patients initiated on category one regimen of directly observed treatment short course (DOTS) at 24 DOTS centers ( $N=156$ ) in E-ward of Mumbai Municipal Corporation (MMC) were interviewed using pre-tested semi-structured schedule, which was designed to elicit the TSB and factors associated with treatment delays. Results: Median duration of cough with expectoration before consulting a provider was 8 weeks ( $\min =1$, $\max =96$ weeks). Risk factors for patient delay were age $<45$ years, living without family. Mean provider delay was 17.91 (SD $=18.026$, range $=7-99$ days). First approach to the private sector for the treatment was associated with provider delay. Mean delay to start continuation phase $(C P)$ was 18.46 days $(S D=16.292)$ ( $\min =4$ days, $\max =67$ days). Delay to go for sputum microscopy, to collect reports, delay in reporting, supposed that treatment is completed as felt better were the reasons to delay to start CP. It was associated with migration, smoking, alcoholism, living away from family, social stigma involving hiding the disease from spouse and relatives. Conclusion: Risk factors

Priya Y. Kulkarni, Atul D. Kulkarni', Sulabha V. Akarte², Jitendra S. Bhawalkar, Devidas T. Khedkar

Departments of P S M, Dr. D Y Patil Medical College, Pimpri,

Pune, ${ }^{1}$ Dr. D Y Patil Medical College, Nerul, Navi Mumbai, ${ }^{2}$ Grant Medical College, Byculla,

Mumbai, Maharashtra, India

Address for the Correspondence:

Dr. Priya Y. Kulkarni, 5, Shanti Sadan, Tarte Colony, Erandawane, Pune - 411 004, Maharashtra, India. E-mail: dr_pdkulkarni@ yahoo.co.in

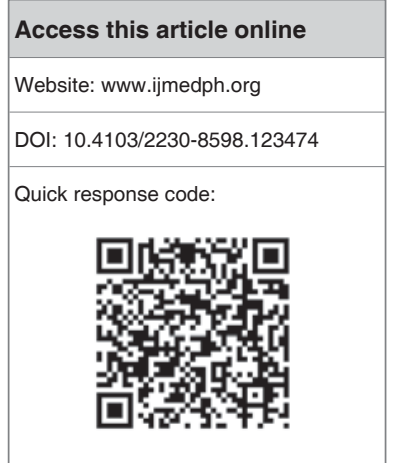
identified for treatment delays were age $<45$ years, lack of family support, first approach to the private sector, migration, addictions and social stigma. Strengthening Revised National TB Control Program by operational research to involve the private sector, improving transfer procedures for migrants, creating public awareness to remove stigma and transport of patients and/or their sputum samples from DOTS centers to microscopy centers may be considered to decrease treatment delays.

Key words: NSP pulmonary tuberculosis, treatment delays, treatment seeking behavior

\section{INTRODUCTION}

India accounts for more than $25 \%$ of the world's incident tuberculosis (TB) cases. ${ }^{[1]}$ New sputum positive (NSP) case detection rate reported by India's Revised National TB Control Program (RNTCP) is above the global targets for TB control. However, it is based on passive case finding, ${ }^{[2]}$ NSP TB patients may remain hidden in the community for prolong time delaying initiation of proper antituberculosis treatment (ATT).

NSP TB patients are the main source of TB infection to the community. ${ }^{[3]}$ Their treatment delays not only increase TB transmission, but also can contribute to development drug resistance. ${ }^{[5]}$ Hence, to study their treatment seeking behavior $(\mathrm{TSB})^{[4]}$ and to identify determinants of treatment delays is important for TB control. ${ }^{[8]}$

Treatment delays can be in the form of patient delay, provider delay ${ }^{\left[{ }^{[6]}\right.}$ and after initiation of ATT, there can be a delay to start continuation phase (CP).

Studies have identified factors associated with treatment delays as lack of awareness, social stigma and preference to consult the private health facilities,. However, re-assessment is required to identify change over time. ${ }^{[6,7]}$ Also, hardly any study reported risk factors for delay to start continuation phase (CP). 